\title{
1
}

\section{Modern Approaches to Radio Network Modelling and Planning}

\author{
Maciej J. Nawrocki, Mischa Dohler and A. Hamid Aghvami
}

The 3rd Generation (3G) Universal Mobile Telecommunications System (UMTS) radio access network relies upon novel, more flexible and efficient communication methods, a consequence of which is that novel modelling and planning approaches become of prime importance to the network's rollout success. In this chapter, we will briefly consider the historical developments of radio network modelling and planning, thereby highlighting the need for a more modern approach to the subject. Equally importantly, we alert the reader to the limitations of modelling tools. The chapter concludes with a discussion of the advantages, disadvantages and limitations of both manual and automated optimisation processes.

\subsection{HISTORICAL ASPECTS OF RADIO NETWORK PLANNING}

One of the co-editors was working as a radio network planning consultant for one of the emerging UMTS networks in the late 1990s. It soon emerged that his point of view on network planning for 3G was surprisingly different from that of the operator's engineers. A likely explanation for this would be that neither party, at that time, had had any practical experience of UMTS network planning. On the one hand was an academic UMTS background and on the other was the operators' extensive GSM network planning experience. Once discussion started among both parties, it turned out that both sides had very different points of view on virtually all network planning aspects, because ... they simply viewed the network using very different planning parameters. While the more academic approach recognised the multitude of parameters influencing a UMTS radio network, the concern of the operators was more the appropriate selection of $3 \mathrm{G}$ base site locations and their static configuration. The operators' view was largely driven by the suggestion of some consultants that the main issue in $3 \mathrm{G}$ network planning was capacity and coverage, which, because no real planning tools were then available, were derived either analytically or using manual measurement regimes.

Understanding UMTS Radio Network Modelling, Planning and Automated Optimisation Edited by Maciej J. Nawrocki, Mischa Dohler and A. Hamid Aghvami (C) 2006 John Wiley \& Sons, Ltd 
So what, really, is network planning? And, is network optimisation part of network planning or is this included in network optimisation? This book is going to give the answers to the above questions and, hopefully, to many more. As will also become apparent, neither the academic nor the operators' initial approach was right or wrong. Without going into too much detail, let us dwell upon a couple of issues related to network planning and optimisation.

Let us start with the choice of an appropriate base site location. Traditionally, the choice has been to locate base sites at the intersects of a triangular grid, thereby covering hexagonally shaped cells. There are many reasons why this approach has been successfully applied to network planning problems, the main reason being its analytical simplicity. Indeed, such a cell-layout uniformly covers any flat surface and is also well approximated by omnidirectional base site antenna elements. The choice of a hexagonal cell shape is further justified when three-sector sites are being used; or was it the hexagonal shape which stipulated the use of three sectors? Either answer certainly contains elements of the truth; however, it is worth pondering on the usefulness of the hexagonal assumption. Indeed other cell layouts are equally possible and are used, as e.g. rectangular grids in Point-to-Multipoint (PMP) systems such as Local Multipoint Distribution Systems (LMDS) (see Chapter 17). Perhaps surprisingly, it turns out that when applied to cellular system, square-shaped cells may also constitute a good modelling approach, which is facilitated by the horizontal plane antenna characteristics (assuming $90^{\circ}$ sectors).

Regular shapes, be they hexagonal or square, largely result from the prior assumption of an homogeneous propagation environment. However, once one looks into the real propagation behaviour, the beauty of regular cell shapes has, sadly, to be abandoned. In the radio propagation community, it is widely known that propagation conditions in urban areas can destroy any regular cell and/or frequency plan, as street canyons become strong ducts for interference which far exceeds the levels anticipated using regular planning approaches. Hexagonality is also lost when the traffic becomes severely nonuniform, something observable not only in CDMA networks but also in any kind of cellular network, albeit in different forms. So, does the choice of medium access technique really impact the cell shapes, site locations and the network planning process as a whole? It definitely does.

This brings us to the next related issue, that of access technique, which is the primary driver for the choice of network planning and optimisation techniques; generally, we distinguish among three mainstream techniques in mobile systems: Frequency Division Multiple Access (FDMA), Time Division Multiple Access (TDMA) and Code Division Multiple Access (CDMA). They guarantee that competing users access the wireless medium in an ideally non-interfering manner, where access is granted in time, frequency or code. For instance, FDMA enables the simultaneous and interferencefree use of a given frequency band by many users, where each user is assigned a sub-part of the entire band. In TDMA, users are assigned orthogonal time slots and in CDMA distinct spreading codes. In summary, access techniques facilitate an optimal use of available resources and their flexible management. To increase flexibility, hybrids of these techniques are desirable and, indeed, widely used in currently deployed communication systems.

First generation networks were using pure FDMA as a fairly inflexible access technique. The basics of network planning consisted of the aggregation of cells into clusters with each cell in the cluster having a unique frequency allowing the proper allocation of frequencies to the whole network. The system performance was limited by the level of inter-cell interference and interference sources were located far from the victim cell. Transmission over a particular link was possible if the signal-tointerference ratio (SIR) was greater than a given threshold, but this lead to an overhead in transmission power resulting in a non-optimal use of spectrum and power resources. The first automated solutions for network planning were developed to address this, most notably the Automatic Frequency Planning (AFP) approach [1] which assured good network quality, given appropriate site location selection and antenna configuration.

The planning and optimisation situation did not change greatly with the introduction of second generation $(2 \mathrm{G})$ networks. They were, of course digital, thereby easing the stringent requirements on signal-to-interference ratio when compared with the analogue predecessor. Although they were still 
heavily reliant on FDMA, advances in technology enabled hybrid extensions to the time domain, thereby leading to more flexible FDMA/TDMA networks (e.g. GSM, IS-136); some systems also started to utilise the time domain more intensively (e.g. DECT, PHS). For these $2 \mathrm{G}$ systems, new concerns in network planning emerged: synchronisation proved to be an important issue, as well as dynamic channel allocation in conjunction with interference avoidance in both the time and frequency domains; however, frequency planning which takes into account frequency hopping (FH) remained the main issues for GSM-like systems [1].

The exception to 2G TDMA/FDMA developments was the North-American IS-95 standard, which was the first commercial cellular system based on CDMA technology. Although it was also a $2 \mathrm{G}$ system, network planning here drifted into new and unknown areas, not all of which were identified or appreciated, despite $3 \mathrm{G}$ being solely dependent on CDMA technology. CDMA, conceptually an excellent idea in which all mobiles use the same frequency band at all times differentiated only by code and power level, requires numerous practical problems to be solved before the network can be referred to as efficient. This process may take years to evolve which only automated solutions can accelerate.

So, what are the problems from a network planning perspective with CDMA in UMTS? To begin with, CDMA based networks are very sensitive to traffic distributions and traffic volumes. Such traffic dependency does not exist in this form in FDMA and/or TDMA based systems and the network for these systems can be simply characterised, e.g., by the capacity (here referred to as hard capacity) regardless of the traffic in the network. In contrast, the 3G system performance depends heavily on the traffic conditions, making many performance measures soft (e.g. soft capacity, soft handoff). Consequently, the network planning engineer cannot plan the network a priori to find a generally optimum solution, because any solution is valid only for a given traffic condition; when the traffic changes, the optimal plan also changes. This demands, at least from a theoretical point of view, the use of automated procedures which attempt dynamically to adapt network parameters to the (near) instantaneous traffic distribution (see Chapter 16).

All of the above effects are amplified by the re-use of the same frequency in every cell, i.e. in all neighbouring cells. Therefore, the coordination distance based planning, which hexagonal and other grid design relies upon, cannot be used for CDMA networks in the same sense as for FDMA based networks; most established methods of cellular network design thus prove useless. Furthermore, a characteristic of CDMA based networks is that the SIR needs to be equal to a given threshold; this is in contrast to FDMA requirements where the SIR should be greater then a threshold and where energy and spectrum use are less than optimal. The strong influence of transmission powers and the resulting interference in CDMA systems make the system performance dependent not only on the traffic level in a given cell, but also on that in neighbouring and further cells; the system is said to be interference limited. Since interference is the prime concern in CDMA networks, the process of network planning attempts to minimise this occurring across all cells simultaneously. Such a planning process is the main topic of this book and it is not an easy task since dynamically interdependent interference occurs virtually everywhere in the network. Indeed, as is now well recognised, it is relatively easy to make a CDMA network operate, but to make it operate well is extremely difficult.

\subsection{IMPORTANCE AND LIMITATIONS OF MODELLING APPROACHES}

The planning of any modern radiocommunication network is carried out by means of computer algorithms, which numerically calculate the desired network characteristics and thereby assist the network planning engineer during the design process. To evaluate and compute any of these characteristics, a model of the physical surroundings and user behaviour must be made available. Such a model ought to be as precise as possible, where the modelling quality determines the consistency and applicability 
of the obtained results to the real world network deployment; an imprecise modelling renders any planning and optimisation approach, no matter how precise, useless.

Because of its importance, Part II of this book is dedicated to those modelling aspects which are crucial in UMTS network planning and optimisation. Each network operates within a given environment which must be reflected as precisely as possible in a proper model. Terrain topography as well as building layout in urban areas constitutes an important part of many network planning solutions. Digital Elevation Models (DEM) of the terrain within which the network operates need to be provided, not only for planning purposes but also to fulfil international procedures, such as cross-border coordination. Models are needed for a plethora of radio communication systems, ranging from TV broadcast systems to cellular and microwave point-to-point systems. In addition to the DEM, modelling of urban environments is also crucial for network planning; this is usually accomplished by means of clutter maps or, in more detailed approaches, through 3D building maps. All these models are a vital prerequisite for another part of the virtual modelling environment used by modern network planners - propagation modelling.

The propagation modelling process might appear to be the easiest of the modelling components, since all phenomena of electromagnetism are well quantified and appropriate approximations to Maxwell's equations readily applicable. In reality, however, propagation models are likely to be the most inaccurate part of this virtual modelling world. This may be for many reasons, the inaccuracy of terrain and building models being the important one. Building models can have errors in vertices in both the horizontal and vertical directions and, even when a 3D map has a high resolution, these errors can be significant compared with reality. Resolution and accuracy do not mean the same thing. Unfortunately, today's scanning technology facilitates only the modelling of general building geometries without precisely taking into account windows, roof shapes, gutters, vegetation, surface roughness etc. Even if a higher precision were possible, the electrical parameters of all the surroundings are generally unknown. Furthermore, statistical propagation modelling, whilst useful for evaluating performance tendencies, is of little use in given real-world network environments.

For a moment, let us consider that we have managed to obtain very high resolution maps of geometrical and electrical parameters describing the urban environment of our network roll-out area. A non-negligible problem soon arises, apart from the cost of this kind of map, how are we to process this huge amount of data? Advances in modern computing technology are breathtaking, but it is still not enough for efficient and accurate propagation computations. Operators are happy today when the standard deviation of an error in propagation prediction is about $8 \mathrm{~dB}$; but, from a link budget perspective, $8 \mathrm{~dB}$ can easily mean a doubling of the communication range! Of course large supercomputers can be employed for this kind of modelling work, but the associated costs are not justified. Paradoxically, although computing power increases dramatically every year, the systems we want to model and simulate also become more complicated. It seems that solutions for propagation modelling will rely on reasonable simplifications for a long time to come, where the degree of simplification will slowly be reduced over the forthcoming years.

Temporal changes to the propagation environment are another important factor which make propagation models inaccurate. Such temporal variations are very difficult to account for; just imagine, e.g., the changes of vegetation throughout the seasons or changes in electrical parameters of the ground and walls after the rain. We have known of many situations where the network was well planned and optimally functioning in the summer, but when winter came and leaves disappeared from the trees, the call drop probability increased significantly because interfering sites became visible in hilly terrain (so-called 'boomer' cells). In addition to this, digital maps should be regularly updated to ensure that all newly constructed buildings are included.

An important part of the entire modelling process relates to users' behaviour. Users can have, e.g., various preferences in service usage or the financial budget allocated for mobile phone calls. These effects are difficult to measure by the operator and are more appropriate to sociological or psychological research than engineering studies. Even if mathematical models of users' behaviour can 
be made (of any aspect), obtaining realistic values for such a model parameters might be impossible. User density and mobility as well as their temporal changes during the day or week are also hard to capture. All these issues create uncertainty in UMTS planning, where the network performance heavily depends on the traffic.

The modelling of the system and network behaviour relies in one way or another on all the constituent models. There are a number of components and phenomena which must be modelled with the highest possible precision, such as antennas, receivers, transmitters, access techniques, codecs, actions taken in the whole Radio Resource Management (RRM) domain and many more. The modelling can precisely reflect transceiver structures and channel variations, making direct use of link-level modelling approaches, or be more general and use more system-level modelling approaches; dynamic effects can be simplified or passed over into static or dynamic system-level simulators. An ideal solution would clearly be to have a full dynamic simulator which includes complete link level models; this is the dream of many researchers and engineers, but some time still must pass before computing solutions enable simulations of every detail for every user within a network with thousands of users within reasonable computing times.

From this brief overview, the question arises of how far we should go into the modelling of the surrounding world for cellular network planning? Interesting considerations related to this concern are presented in [2]. There is also one particular trend that has become visible in the past few years: if reality cannot be modelled precisely enough so that errors and simplifications do not impair the planning results, use the reality instead - this makes measurements increasingly important, not only for network planning validation but also as an input to the network planning and optimisation processes.

In summary, this section, albeit lacking in technical depth, serves to emphasise the various modelling issues that are important to the radio network planning process and hence to the level of service satisfaction experienced by the network users.

\subsection{MANUAL VERSUS AUTOMATED PLANNING}

The models from Section 1.2 form the basis for the simulation software used by network planning engineers to dimension and plan the network. Such radio communication system design requires highly sophisticated computer aided design (CAD) tools. These tools facilitate refined network planning, the complexity of which is generally beyond the scope of any manual approach. Planning tools and optimisation tools must be distinguished. A planning tool consists of a database, a Geographical Information System (GIS), propagation modules etc., and is used to predict the network performance for a given set of system (analysis). An optimisation tool takes this data and tries to find such system parameters as will achieve the best network performance (synthesis, aided by analysis; see Chapter 13).

Radio network planning can be defined as designing a network structure and its configuration to meet certain quality requirements. There may be a number of criteria defined by the operator, and usually these differ from operator to operator. The criteria can take into account such topics as coverage, Quality of Service (QoS), equipment and other costs, revenues from network operation (see Chapters 7 and 8 ) etc. and can be used to verify the network quality either by the engineer (manual decision) or by the software (automated decision). A schematic presentation of the modelling, planning and optimisation tasks in the network design process is shown in Figure 1.1.

Having a new generation network to deploy, operators start from some simple planning rules (manual planning) to get acquainted with the system behaviour. The first networks are usually rather badly planned since the transition to any new technology requires some time to understand the relationships between any change of configuration and the response in the network; such a testing approach is typically applied to trials prior to the official network launch. Under these simplified operational conditions, the operator is usually unable to learn and recognise all the aspects of running the network since there is usually insufficient traffic demand to experience extreme load conditions. When the 


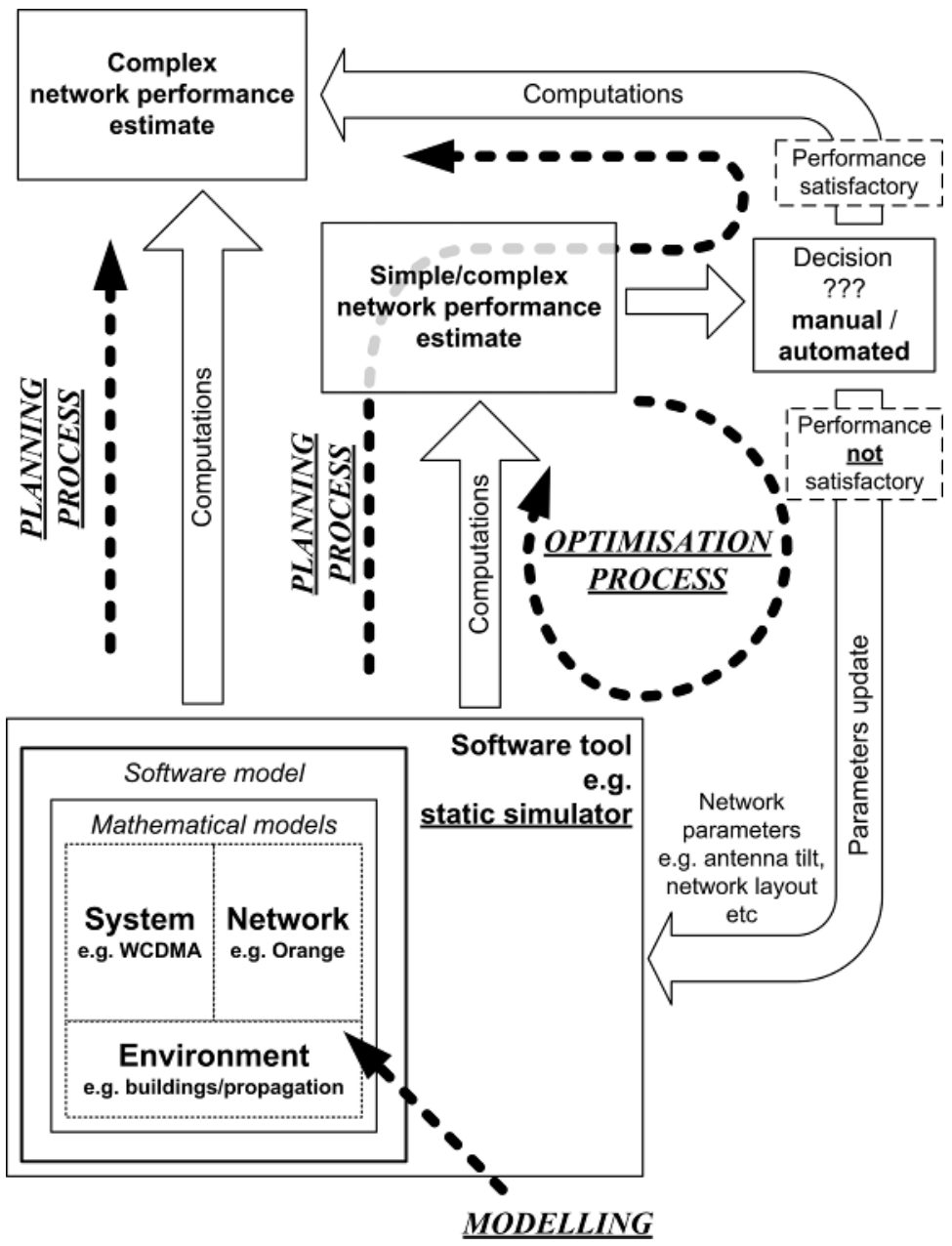

Figure 1.1 Modelling, planning and optimisation functions in the network design process.

network starts its commercial operation, however, the traffic increases and the operator faces new situations in which the system cannot serve the increased traffic; this requires some changes to the network configuration and is usually accomplished by means of optimisation. It must be noted that the term optimisation is sometimes misunderstood in the context of optimisation being part of the pre-launch network planning. From an operational network point of view, and the definition proposed at the beginning of this section, the optimisation process should be treated as part of the ongoing network planning and all these activities should be performed over the whole network operation period, e.g. perhaps for 10 years or more. Historically, some operators have had two separate departments, one for planning and another for optimisation; however, this type of operation has many times proved inefficient, thus partially answering the above dilemma.

With an ever increasing complexity of real networks, planning and optimisation requires the use of sophisticated software tools. Their sole task is to assist the planning engineer and help him or her process the enormous amount of relevant information into a comprehendible form. Such a process is 
usually accomplished by means of a number of modules within a given planning tool, e.g. a database, the GIS, propagation and CDMA Monte-Carlo simulation modules. Optimisation tools are even more important in this than planning tools since they use automated search processes on good initial network designs, something beyond human ability. Although modern software solutions are able to deliver a full network design by means of a single mouse click if this is accepted unchecked, there are risks. The uncritical acceptance of information received from planning or optimisation tools is indeed very dangerous; we have known of cases where inferior tools were preferred simply because they required less involvement of the radio design engineer. It should be recognised that CAD software is used to help people, but not as a substitute for them. Tools should be user friendly, accurate, fast and flexible (ease of connecting third-party modules) and allow group working on a common project.

In summary, the ideal situation would be to encourage experienced planning engineers to use automated software in the initial network planning and optimisation stages, but to rely on their own intuition and experience in the final network verification stage.

\section{REFERENCES}

[1] http://www.datax.pl.

[2] Trevor Gill, 'Radio planning and optimisation - the challenge ahead', 4th International Conference on 3G Mobile Communication Technologies, London, 25-27 June 2003, pp. 28-30. 
